MATHEMATICS OF COMPUTATION

Volume 74, Number 249, Pages 383-388

S 0025-5718(04)01676-X

Article electronically published on May 21, 2004

\title{
ON THE ABSOLUTE MAHLER MEASURE OF POLYNOMIALS HAVING ALL ZEROS IN A SECTOR. II
}

\author{
GEORGES RHIN AND QIANG WU
}

\begin{abstract}
Let $\alpha$ be an algebraic integer of degree $d$, not 0 or a root of unity, all of whose conjugates $\alpha_{i}$ are confined to a sector $|\arg z| \leq \theta$. In the paper On the absolute Mahler measure of polynomials having all zeros in a sector, G. Rhin and C. Smyth compute the greatest lower bound $c(\theta)$ of the absolute Mahler measure $\left(\prod_{i=1}^{d} \max \left(1,\left|\alpha_{i}\right|\right)\right)^{1 / d}$ of $\alpha$, for $\theta$ belonging to nine subintervals of $[0,2 \pi / 3]$. In this paper, we improve the result to thirteen subintervals of $[0, \pi]$ and extend some existing subintervals.
\end{abstract}

\section{INTRODUCTION}

Let $P(z) \neq z$ be a monic polynomial with integer coefficients, irreducible over the rationals, of degree $d \geq 1$, and having zeros $\alpha_{1}, \cdots, \alpha_{d}$. Its relative Mahler measure $M(P)$, given by

$$
M(P)=\prod_{i=1}^{d} \max \left(1,\left|\alpha_{i}\right|\right)
$$

is either 1 (if $P$ is cyclotomic) or thought to be bounded away from 1 by an absolute constant (if $P$ is not cyclotomic) [B1], [B2]. When the zeros of $P$ are restricted to a closed set $V$ which does not contain the whole unit circle, however, one can say much more. Then, from a result of Langevin $\left[\mathrm{LA}\right.$ there is a constant $C_{V}>1$ such that the absolute Mahler measure $\Omega(P):=M(P)^{1 / d}$ for such $P$ is either 1 or else satisfies

$$
\Omega(P) \geq C_{V}
$$

So we try to find the largest value for the constants $C_{V}$ when $V$ is the sector $\{z:|\arg z| \leq \theta\}$, where $0 \leq \theta<\pi$. We denote this best value by $c(\theta)$. It is clear that $c(\theta)$ is a nonincreasing function of $\theta$ and, using the polynomials $z^{2 k+1}-2$ as $k \rightarrow \infty$, that $c(\theta) \rightarrow 1$ as $\theta \rightarrow \pi$.

In a previous paper [RS], G. Rhin and C. Smyth succeeded in finding $c(\theta)$ exactly for $\theta$ in nine intervals. They conjectured that $c(\theta)$ is a "staircase" function of $\theta$ which is constant except for finitely many left discontinuities in any interval $[0, \Theta)$ for $\Theta<\pi$. They used auxiliary functions of the type

$$
f_{i}(\theta)=\left\{\max _{z \in W_{\theta}}\left|z^{a_{i}} \prod_{j} P_{i j}(z)^{e_{i j}}\right|\right\}^{-1 /\left(2 a_{i}+\sum_{j} e_{i j} \operatorname{deg} P_{i j}\right)}
$$

Received by the editor March 12, 2003 and, in revised form, August 10, 2003.

2000 Mathematics Subject Classification. Primary 11R04, 12D10.

(C)2004 American Mathematical Society 
in the sector $W_{\theta}=\{|z|<1,|\arg z| \leq \theta\}$. Then they find:

Theorem. There is a continuous, monotonically decreasing function $f(\theta)>1$ for $0 \leq \theta \leq 2 \pi / 3$ and there is a staircase function $g(\theta)>1$ such that

$$
\min (f(\theta), g(\theta)) \leq c(\theta) \leq g(\theta) \quad(0 \leq \theta<\pi) .
$$

The function $f(\theta)$ is given by $f(\theta):=\max _{i=1}^{9} f_{i}(\theta)$. The function $g(\theta)$ is a decreasing staircase having left discontinuities at the angles given (in degrees) in Table 4 of [RS. The corresponding absolute measure is the new smaller value of $g(\theta)$ which is the smallest value of $\Omega(P)$ that could be found, for $P$ having all its zeros in $|\arg z| \leq \theta$.

In the proof of the Theorem, Rhin and Smyth referred to Langevin's proof $\underline{\mathrm{LA}}$, which has three basic ingredients:

(i) the observation that the set $V_{1}=V \cap\{z \in \mathbb{C}:|z| \leq 1\}$ has transfinite diameter less than 1 ,

(ii) a result of Kakeya to the effect that for any set $W$ of transfinite diameter less than 1 and symmetric about the real axis there is a nonzero polynomial $A$ with integer coefficients such that $\sup _{z \in W}|A(z)|<1$,

(iii) deduction of $\Omega(P) \geq C_{V}$ from (i) and (ii) using $W:=\{z: z \in V$ and $\bar{z} \in V\}$.

For the computation of $f(\theta)=\max _{i=1}^{9} f_{i}(\theta)$, they use, for each $f_{i}$, an auxiliary polynomial $A$ as in (ii), and they choose such $A$ of the form $z^{a} R(z)$, where $a$ is a positive integer and $R$ is a reciprocal polynomial of degree $r$ with integer coefficients, i.e.,

$$
A(z)=z^{a} \prod_{j} P_{j}(z)^{e_{j}}
$$

The function

$$
m(\theta)=\sup _{z \in W_{\theta}}|A(z)|^{\frac{1}{2 a+r}}
$$

is then associated with $A$. Then Langevin's argument of (iii) above gives

$$
\Omega(P) \geq \frac{1}{m(\theta)} \quad \text { if } \operatorname{gcd}(P, A)=1
$$

for $P$ irreducible, of degree $d$, with integer coefficients. For, if $\alpha_{1}, \cdots, \alpha_{d}$ are the zeros of $P$, then, since $R(z)=z^{r} R\left(z^{-1}\right)$, one has

$$
\begin{aligned}
1 & \leq\left|\prod_{i=1}^{d} \alpha_{i}^{a} R\left(\alpha_{i}\right)\right|=\prod_{\left|\alpha_{i}\right| \leq 1}\left|\alpha_{i}^{a} R\left(\alpha_{i}\right)\right| \times \prod_{\mid \alpha_{i}>1}\left|\alpha_{i}^{a+r} R\left(\alpha_{i}^{-1}\right)\right| \\
& =\prod_{\left|\alpha_{i}\right| \leq 1}\left|\alpha_{i}^{a} R\left(\alpha_{i}\right)\right| \times \prod_{\left|\alpha_{i}\right|>1}\left|\left(\alpha_{i}^{-1}\right)^{a} R\left(\alpha_{i}^{-1}\right)\right| \times \prod_{\left|\alpha_{i}\right|>1} \alpha_{i}^{2 a+r} \\
& \leq m(\theta)^{(2 a+r) d} M(P)^{2 a+r}
\end{aligned}
$$

whence $\Omega(P) \geq 1 / m(\theta)$.

Then each $f_{i}(\theta)$ was defined, as in equation (1), to be the function $1 / m(\theta)$ corresponding to a polynomial $A$ chosen so that $f\left(\theta_{i}\right)>g\left(\theta_{i}\right)$ and so that the length of the interval $\left[\theta_{i}, \theta_{i}^{\prime}\right]$ over which $f(\theta)>g(\theta)$ was as long as possible. Thus, if $g\left(\theta_{i}\right)=\Omega\left(P_{*}\right)$ (Table 4 in [RS] , then $\Omega\left(P_{*}\right)<f_{i}\left(\theta_{i}\right)$. From (2) it follows that $P$ is a factor of $A$ and that, among polynomials with all conjugates in $|\arg z| \leq \theta_{i}$, only factors of $A$ can have absolute measure less than $f_{i}\left(\theta_{i}\right)$. Now $P_{*}$ does indeed divide 
$A$, and in fact it has the smallest absolute measure among factors $A$ of measure $>1$. It follows that $\Omega\left(P_{*}\right)$ is the smallest value of the absolute measure for polynomials having all zeros in $|\arg z| \leq \theta$ for $\theta \in\left[\theta_{i}, \theta_{i}^{\prime}\right]$. Hence, $c(\theta)=\Omega\left(P_{*}\right)$ for these $\theta$.

One of the main problems in the previous paper was to find for each interval suitable polynomials to use to obtain a good auxiliary function. In fact they only used a heuristic process and produced a table of good $P_{j}$ which were for almost all polynomials of one of the following six types:

$$
\begin{array}{lll}
z^{n} Q\left(z+z^{-1}-k\right) & (k=3,2,1,0) & (\text { types } 1,2,3,4), \\
z^{n} S\left(z+z^{-1}-2\right) & \text { where } S(x)=Q(1) x^{n} Q(1+1 / x) & (\text { type } 5), \\
z^{n}(Q(z)+Q(1 / z)) & & \text { (type 6). }
\end{array}
$$

Here $Q$ is a degree $n$ monic polynomial with small coefficients, also with $Q(1)= \pm 1$ for the fifth type. As pointed out in [RS p. 301] "The reason for polynomials of these types giving good polynomials appears mysterious, however."

The second author gave in [WU] an algorithm improving the ones given by $\mathrm{P}$. Borwein and T. Erdelyi $[\mathrm{BE}]$ and L. Habsieger and B. Salvy [HS] to find polynomials which have to be involved in such auxiliary functions $f_{i}(\theta)$. This method gives better lower bounds for $c(\theta)$ for four new intervals of $\theta$ between 0 and $7 \pi / 9$.

Table 1 shows the 13 intervals $\left[\theta_{i}, \theta_{i}^{\prime}\right]$ where $f(\theta)>g(\theta)$, so that $c(\theta)=g(\theta)=$ $g\left(\theta_{i}\right)$ for $\theta$ in those intervals; i.e., $c(\theta)$ is known exactly. Here $c(\theta)=c\left(\theta_{i}\right)=\Omega(p)$ for $\theta \in\left[\theta_{i}, \theta_{i}^{\prime}\right]$. The fifth column presents the results from $[\overline{\mathrm{RS}}]$. The polynomial $P$ is read off from Table 3. The function $f(\theta)$ is given by $f(\theta):=\max _{i=1}^{13} f_{i}(\theta)$ where the $f_{i}(\theta)$ are defined as in (1) and the $a_{i}, P_{i j}$ and the $e_{i j}$ are given by Table 2, using the polynomials of Table 3 The function $g(\theta)$ employs the polynomials listed in Table 4 of [RS], where we add the polynomials $P_{25}$ and $P_{31}$ of our Table 3 .

Table 2 gives the auxiliary functions

$$
A_{i}(z)=z^{a_{i}} \prod_{j} P_{i j}(z)^{e_{i j}}
$$

used to compute $f_{i}(\theta)$ for $i=1, \cdots, 13$.

Table 3) shows the reciprocal polynomials used in Tables[1]and2, where $d=\operatorname{deg} P$ and $\varphi(P)=\max \{|\arg z|: P(z)=0\}$.

TABLE 1.

\begin{tabular}{|l|r|r|r|r|l|}
\hline$i$ & $c(\theta)$ & $\theta_{i}$ & $\theta_{i}^{\prime}$ & $\theta_{i}^{\prime}$ in $[\mathrm{RS}]$ & $P$ \\
\hline 1 & 1.618034 & 0.000000 & 17.40 & 17.39 & $P_{2}$ \\
2 & 1.539222 & 26.408740 & 26.65 & 26.65 & $P_{7}$ \\
3 & 1.493633 & 30.440145 & 30.74 & 30.59 & $P_{8}$ \\
4 & 1.303055 & 47.941432 & 49.46 & 49.46 & $P_{9}$ \\
5 & 1.300734 & 50.830684 & 50.96 & & $P_{12}$ \\
6 & 1.259269 & 60.890196 & 63.87 & 63.87 & $P_{15}$ \\
7 & 1.210608 & 73.631615 & 74.04 & 73.99 & $P_{19}$ \\
8 & 1.154618 & 80.241034 & 82.43 & 81.40 & $P_{21}$ \\
9 & 1.129338 & 86.708519 & 91.40 & 91.40 & $P_{23}$ \\
10 & 1.096504 & 101.353607 & 101.99 & & $P_{25}$ \\
11 & 1.055423 & 112.647119 & 115.32 & 115.32 & $P_{29}$ \\
12 & 1.033097 & 127.355699 & 129.47 & & $P_{31}$ \\
13 & 1.020306 & 137.102805 & 137.15 & & $P_{37}$ \\
\hline
\end{tabular}


TABLE 2 .

\begin{tabular}{|c|c|c|c|c|}
\hline$i$ & $\theta_{i}^{\prime}$ & Polynomials $P_{i j}$ & $\overline{\text { Exponents } e_{i j}}$ & $a_{i}$ \\
\hline 1 & 17.40 & $\begin{array}{lllll}P_{1} & P_{2} & P_{3} & P_{4} & P_{5}\end{array}$ & 2102105610000540014000258 & 20829 \\
\hline 2 & & & & \\
\hline 3 & & $P_{1} \quad P_{8}$ & & \\
\hline 4 & & $\begin{array}{llll}P_{1} & P_{9} & P_{12} & P_{14}\end{array}$ & 40064213732 & \\
\hline 5 & & $\begin{array}{lllllll}P_{1} & P_{9} & P_{10} & P_{11} & P_{12} & P_{13} & P_{14}\end{array}$ & 1585901071002670 & 11684 \\
\hline 6 & & $\begin{array}{lllll}P_{1} & P_{14} & P_{15} & P_{16} & P_{18}\end{array}$ & 21818 & 958 \\
\hline 7 & & $P_{10} P_{0}$ & 065 & 12853 \\
\hline & & & & \\
\hline & & $\begin{array}{lllllll}P_{1} & P_{14} & P_{21} & P_{23} & P_{24} & P_{28}\end{array}$ & 300 & 12165 \\
\hline 10 & & 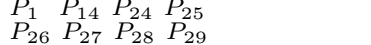 & $\begin{array}{ll}3 & 10 \\
3 & 0\end{array}$ & 10268 \\
\hline 11 & & $\begin{array}{llllll}P_{1} & P_{14} & P_{24} & P_{28} & P_{29} & P_{30}\end{array}$ & 039730 & \\
\hline 12 & & $\begin{array}{llllll}P_{1} & P_{14} & P_{24} & P_{28} & P_{30} & P_{31} \\
P_{32} & P_{33} & P_{34} & P_{35} & P_{38} & \end{array}$ & $\begin{array}{llllllll}01916 & 03282 & 02376 & 02271 & 03763 & 01257 \\
03267 & 00301 & 00159 & 00756 & 00641\end{array}$ & 10725 \\
\hline 13 & 137.15 & $\begin{array}{llllll}P_{1} & P_{14} & P_{24} & P_{28} & P_{30} & P_{32} \\
P_{35} & P_{36} & P_{37} & P_{38} & P_{39}\end{array}$ & $\begin{array}{lllllll}01491 & 03082 & 01982 & 01696 & 02448 & 01997 \\
01777 & 00576 & 00770 & 01624 & 00323 & \end{array}$ & 15026 \\
\hline
\end{tabular}

TABLE 3 .

\begin{tabular}{|c|c|c|c|c|c|c|c|c|c|c|c|c|}
\hline$P$ & $\Omega(P)$ & $\varphi(P)$ & $\mathrm{d}$ & & lest & If of & oefficie & $\mathrm{s}$ of $P$ & & & & \\
\hline$P_{1}$ & 1.000000 & 0.000000 & 2 & $\overline{1}$ & -2 & & & & & & & \\
\hline$P_{2}$ & 1.618034 & 0.000000 & 2 & 1 & -3 & & & & & & & \\
\hline$P_{3}$ & 1.634404 & 17.665834 & 16 & 1 & -25 & 281 & -1873 & 8238 & -25211 & 55246 & -88031 & 102749 \\
\hline$P_{4}$ & 1.610559 & 18.863408 & 8 & 1 & -12 & 58 & -143 & 193 & & & & \\
\hline$P_{5}$ & 1.611995 & 20.717188 & 12 & 1 & -18 & 141 & -628 & 1756 & -3219 & 3935 & & \\
\hline$P_{6}$ & 1.547928 & 26.301669 & 10 & 1 & -14 & 85 & -287 & 585 & -739 & & & \\
\hline$P_{7}^{0}$ & 1.539222 & 26.408740 & 4 & 1 & -5 & 9 & & & & & & \\
\hline$P_{8}$ & 1.493633 & 30.440145 & 6 & 1 & -8 & 26 & -37 & & & & & \\
\hline$P_{9}$ & 1.303055 & 47.941432 & 6 & 1 & -5 & 13 & -17 & & & & & \\
\hline$P_{10}$ & 1.322672 & 49.112713 & 12 & 1 & -11 & 62 & -212 & 487 & -788 & 923 & & \\
\hline$P_{11}$ & 1.312282 & 49.353680 & 4 & 1 & -3 & 5 & & & & & & \\
\hline$P_{12}$ & 1.300734 & 50.830684 & 8 & 1 & -7 & 26 & -53 & 67 & & & & \\
\hline$P_{13}$ & 1.308589 & 52.798885 & 14 & 1 & -12 & 76 & -302 & 832 & -1669 & 2510 & -2871 & \\
\hline$P_{14}^{10}$ & 1.000000 & 60.000000 & 2 & 1 & -1 & & & & & & & \\
\hline$P_{15}$ & 1.259269 & 60.890196 & 6 & 1 & -4 & 10 & -13 & & & & & \\
\hline$P_{16}$ & 1.245865 & 68.365783 & 12 & 1 & -7 & 30 & -85 & 175 & -268 & 309 & & \\
\hline$P_{17}$ & 1.241661 & 72.761003 & 8 & 1 & -5 & 16 & -29 & 35 & & & & \\
\hline$P_{18}^{11}$ & 1.238359 & 73.295530 & 8 & 1 & -4 & 13 & -23 & 28 & & & & \\
\hline$P_{19}$ & 1.210608 & 73.631615 & 6 & 1 & -3 & 7 & -9 & & & & & \\
\hline$P_{20}$ & 1.208398 & 74.983796 & 8 & 1 & -4 & 12 & -21 & 25 & & & & \\
\hline$P_{21}$ & 1.154618 & 80.241034 & 8 & 1 & -3 & 8 & -13 & 15 & & & & \\
\hline$P_{22}$ & 1.189207 & 81.578941 & 4 & 2 & -4 & 5 & & & & & & \\
\hline$P_{23}$ & 1.129338 & 86.708519 & 6 & 1 & -2 & 4 & -5 & & & & & \\
\hline$P_{24}^{23}$ & 1.000000 & 90.000000 & 2 & 1 & 0 & & & & & & & \\
\hline$P_{25}$ & 1.096504 & 101.353606 & 10 & 1 & -2 & 5 & -9 & 12 & -13 & & & \\
\hline$P_{26}$ & 1.106899 & 101.562999 & 6 & 1 & -1 & 2 & -3 & & & & & \\
\hline$P_{27}^{2}$ & 1.101001 & 106.852539 & 12 & 1 & -2 & 6 & -12 & 20 & -26 & 29 & & \\
\hline$P_{28}$ & 1.000000 & 108.000000 & 4 & 1 & -1 & 1 & & & & & & \\
\hline$P_{29}$ & 1.055423 & 112.647119 & 8 & 1 & -1 & 2 & -3 & 3 & & & & \\
\hline$P_{30}$ & 1.000000 & 120.000000 & 2 & 1 & 1 & & & & & & & \\
\hline$P_{31}$ & 1.033097 & 127.355699 & 12 & 1 & -1 & 2 & -3 & 4 & -5 & 5 & & \\
\hline$P_{32}$ & 1.000000 & 128.571429 & 6 & 1 & -1 & 1 & -1 & & & & & \\
\hline$P_{33}$ & 1.040011 & 131.102998 & 10 & 1 & -1 & 2 & -3 & 3 & -3 & & & \\
\hline$P_{34}$ & 1.039015 & 131.327187 & 14 & 1 & 0 & 1 & -2 & 2 & -3 & 3 & -3 & \\
\hline$P_{35}$ & 1.000000 & 135.000000 & 4 & 1 & 0 & 0 & & & & & & \\
\hline$P_{36}$ & 1.034105 & 136.742591 & 10 & 1 & -1 & 2 & -2 & 2 & -3 & & & \\
\hline$P_{37}$ & 1.020306 & 137.102805 & 12 & 1 & 0 & 1 & -1 & 1 & -2 & 1 & & \\
\hline$P_{38}$ & 1.000000 & 140.000000 & 6 & 1 & 0 & 0 & -1 & & & & & \\
\hline$P_{39}$ & 1.000000 & 144.000000 & 4 & 1 & 1 & 1 & & & & & & \\
\hline
\end{tabular}

\section{SEARCH FOR GOOD POLYNOMIALS FOR THE AUXILIARY FUnCTIONS}

Let $\theta<\pi$ be a fixed angle. For a nonzero polynomial $A \in \mathbb{Z}[z]$ we define $a=a(A)$ to be the multiplicity of the root 0 of $A$ and $\|A\|=\sup _{z \in W_{\theta}}|A(z)|$.

As we have seen in the introduction, the search for a good auxiliary function $f$ for $W_{\theta}$ is equivalent to seeking a polynomial $A$ (such that $z^{-a} A$ is reciprocal) in $\mathbb{Z}[z]$ and such that $\|A\|^{1 /(a(A)+\operatorname{deg} A)}$ is as small as possible.

Let $A_{n}$ be the polynomial of degree $n$ such that

$$
\left\|A_{n}\right\|^{\frac{1}{a\left(A_{n}\right)+n}}=\min _{\substack{A \in \mathbb{Z}[z] \\ \operatorname{deg} A=n}}\|A\|^{\frac{1}{a(A)+n}} .
$$


We can define

$$
\tau_{\theta}=\lim _{n \rightarrow \infty}\left\|A_{n}\right\|^{\frac{1}{a\left(A_{n}\right)+n}}
$$

as a generalization of $t_{\mathbb{Z}}\left(W_{\theta}\right)$ which is the integer transfinite diameter of $W_{\theta}$ (in this case the exponent of $\left\|A_{n}\right\|$ is $\left.1 / n\right)$.

Then the factors of the polynomials $A_{n}$ lead to good auxiliary functions as follows. It is difficult to compute the polynomials $A_{n}$ for $n$ large, so we will compute some polynomials $A_{n}^{\prime}$ of sufficiently large degree (say 40 ) where the norm $\left\|A_{n}^{\prime}\right\|$ is sufficiently small and use their factors $Q_{j}$ inside the function $f$. For this we use the following algorithm, which was already described in WU.

Step 1 . We use the LLL algorithm to find a polynomial $Q(x)$ of degree $m$ (say 30 ) in $\mathbb{Z}[x]$ which has a small sup norm in the interval $[2 \cos \theta, 2]$. Then we choose the integer $a$ such that the polynomial $A=z^{a+m} Q(z+1 / z)$ has a norm $\|A\|^{1 /(2 a+2 m)}$ as small as possible.

It is well known that the LLL algorithm gives better results when used in low dimension. So, in Step 2 we will show that $A_{n}^{\prime}$ has an explicit factor of large degree.

Step 2. We use the previous bound and a generalization of the orthogonal MüntzLegendre polynomials to find polynomials that must divide $A_{n}^{\prime}=A$ (where $n=$ $a+2 m)$ in $\mathbb{Z}[z]$.

Step 3. We use now the LLL algorithm to find new polynomial factors of $A_{n}^{\prime}$.

By this algorithm, we find the polynomials $P_{25}$ and $P_{31}$, which not only improve the function $g(\theta)$, but also give better bounds for $c(\theta)$ in the intervals $[101.35,101.99]$ and $[127.35,129.47]$. We also find polynomials (for example $P_{13}$ ) that do not improve the function $g(\theta)$ but give us a new interval in which $c(\theta)$ is known exactly. Furthermore, we find some other polynomials for the auxiliary function which enable us to extend existing intervals.

\section{Computation of the Auxiliary Functions}

We use (for a fixed $\theta$ ) the auxiliary function

$$
f(z)=|z|^{a} \prod_{j=1}^{J}\left|Q_{j}(z)\right|^{e_{j}}
$$

where the polynomials $Q_{j}$ are those which have been computed in Section 2 and such that the positive rationals $a$ and $e_{j}$ satisfy the linear condition

$$
2 a+\sum_{j=1}^{J} e_{j} \operatorname{deg} Q_{j}=1 .
$$

The optimal function $f$ is obtained by semi-infinite linear programming WU], [RS]. This gives four new intervals for $c(\theta)$. Moreover, technical improvements allow us to enlarge some intervals found earlier.

\section{REFERENCES}

[BE] P. Borwein and T. Erdelyi, The integer Chebyshev problem, Math. Comp. 65, (214) (1996), 661-681. MR96g:11077

[B1] D. W. Boyd, Variations on a theme of Kronecker, Canad. Math. Bull. 21 (1978), 129-133. MR 58:5580

[B2] D. W. Boyd, Speculations concerning the range of Mahler's measure, Canad. Math. Bull. 24 (4) (1981), 453-469. MR83h:12002 
[HS] L. Habsieger and B. Salvy, On integer Chebyshev polynomials, Math. Comp. 66 (218) (1997), 763-770. MR97f:11053

[LA] M. Langevin, Minorations de la maison et de la mesure de Mahler de certains entiers algebriques, C. R. Acad. Sci. Paris 303 (1986), 523-526. MR87m:11105

[RS] G. Rhin and C. J. Smyth, On the absolute Mahler measure of polynomials having all zeros in a sector, Math. Comp. 64 (209) (1995), 295-304. MR95c:11123

[WU] Q. Wu, On the linear independence measure of logarithms of rational numbers, Math. Comp. 72 (242) (2003), 901-911. MR2003m:11111

Laboratoire MMAS, CNRS UMR 7122, Université de Metz, Ile du Saulcy, 57045 MeTZ Cedex 1, France

E-mail address: rhin@poncelet.univ-metz.fr

Laboratoire MMAS, CNRS UMr 7122, Université de Metz, Ile du Saulcy, 57045 MeTZ Cedex 1, France

E-mail address: wu@poncelet.univ-metz.fr 